\title{
Multipath Interferometer Polished Microsphere for Enhanced Temperature Sensing
}

\author{
André D. Gomes ${ }^{1,2} \odot$, Fatemeh Karami ${ }^{3}$, Mohammad I. Zibaii ${ }^{3}$, Hamid Latifi ${ }^{3}$, \\ and Orlando Frazão $1,2 \mathbb{C}$ \\ ${ }^{1}$ Centre for Applied Photonics, INESC TEC, Faculty of Sciences, University of Porto, Porto 4169-007, Portugal \\ ${ }^{2}$ Department of Physics and Astronomy, Faculty of Sciences, University of Porto, Porto 4169-007, Portugal \\ ${ }^{3}$ Laser and Plasma Research Institute, Shahid Beheshti University, Tehran 1983969411, Iran
}

Manuscript received January 16, 2018; revised February 16, 2018; accepted March 22, 2018. Date of publication March 26, 2018; date of current version April 19, 2018.

\begin{abstract}
A multipath interferometer based on a polished multimode microsphere is proposed for temperature sensing. Asymmetry was introduced in the structure by polishing the microsphere with a certain angle. A new set of optical paths are generated in the polished microsphere due to the different reflections inside of the structure. The interference between the different optical paths can be seen as the superposition of several two-wave interferometers, which can be discerned through signal processing. Intensity exchange between different components of the interferometer was observed when changing the temperature. The temperature sensitivity is more than three-fold for smaller cavities. Moreover, a sensitivity enhancement is also verified if a correlation method is used. In such a case, the sensitivity to temperature is even higher than just considering smaller cavities.
\end{abstract}

Index Terms-Correlation method, fiber interferometer, microsphere, signal processing, temperature sensing.

\section{INTRODUCTION}

Several different configurations of interferometric cavities have been explored over the years for multiple applications [1]. For instance, high-temperature sensing is one of the great applications of interferometric cavities in optical fibers due to their small size and good thermal strength [2]. Traditional configurations involve placing a diaphragm at the end face of the fiber [3]. However, there are other configurations using different optical fibers or even ablation techniques to produce these structures. For example, a hollow-core silica tube cavity achieved a temperature sensitivity of $8.11 \mathrm{pm} /{ }^{\circ} \mathrm{C}$ for high-temperature sensing [2]. A focused ion-beam milled cavity on a fiber taper tip could obtain a temperature sensitivity of $15.8 \mathrm{pm} /{ }^{\circ} \mathrm{C}$ [4]. A similar sensitivity was reported in etched $\mathrm{P}_{2} \mathrm{O}_{5}$-doped silica fibers postprocessed with focused ion beam to create the cavity [5]. A photonic crystal fiber tip coated with polyvinyl alcohol was explored for temperature sensing, obtaining a sensitivity of $12.8 \mathrm{pm} /{ }^{\circ} \mathrm{C}[6]$.

Microspheres on top of the optical fibers have attracted much attention given the possibility of whispering gallery modes excitation [7]. Microspheres can also be produced to work as interferometric cavities. For example, a hollow-core microsphere on top of a fiber was demonstrated to be a three-wave interferometer and the inline configuration a two-wave interferometer [8]. In terms of sensitivity, the first configuration presented a sensitivity of $1.90 \mathrm{pm} /{ }^{\circ} \mathrm{C}$, and for the inline configuration, a sensitivity of $1.17 \mathrm{pm} /{ }^{\circ} \mathrm{C}$ was reported. An array of silica microspheres was also demonstrated, with a temperature sensitivity of $20.3 \mathrm{pm} /{ }^{\circ} \mathrm{C}[9]$.

Corresponding author: A. D. Gomes (e-mail: ardcgomes@gmail.com). Associate Editor: Xuewen Shu.

Digital Object Identifier 10.1109/LSENS.2018.2819365
In this article, a multipath interferometer based on a polished multimode microsphere is proposed for enhanced temperature sensing. The polished structure creates a new set of optical paths though different reflections inside of the microsphere. The interference between the different optical paths can be seen as the superposition of several two-wave interferometers that can be discerned using adequate signal processing. Temperature sensing can be achieved either by monitoring one of the interferometric components or by using a correlation method.

\section{EXPERIMENTAL SETUP AND RESULTS}

Initially, a microsphere was created at the end of a 15-mm-long cleaved multimode fiber using a fusion splicer Sumitomo Electronics (TYPE-71C) in manual mode. The fiber was a step index multimode fiber with a core/cladding diameter of 50/125 $\mu \mathrm{m}$. The electric arc was centered on the fiber, away from the fiber end. The following parameters were used in the process: $0 \mathrm{~ms}$ prefusion time, $2000 \mathrm{~ms}$ fusion time, and standard arc power +100 (absolute arc-power was not possible to attain, only relative values). Two electric arcs were applied. Before creating the microsphere, the multimode fiber tip reflection spectrum is similar to the one demonstrated by Li et al. [10]. When the microsphere is formed, new interference peaks are formed and a decrease in the signal intensity is observed. The structure was submitted to different temperatures, decreasing from $800{ }^{\circ} \mathrm{C}$ to $400{ }^{\circ} \mathrm{C}$, and the reflection peak around $1560 \mathrm{~nm}$ was monitored. The sensitivity of the multimode microsphere structure to temperature is $12.1 \pm 0.3$ $\mathrm{pm} /{ }^{\circ} \mathrm{C}$, which is slightly lower than just the multimode fiber tip [10].

A similar microsphere was created at the end of a graded-index multimode fiber using a focused $\mathrm{CO}_{2}$ laser. The fiber had a core/cladding diameter of $62.5 / 125 \mu \mathrm{m}$. The geometric characteristics of the microsphere depend on the power, number of pulses, beam shape, and duty 


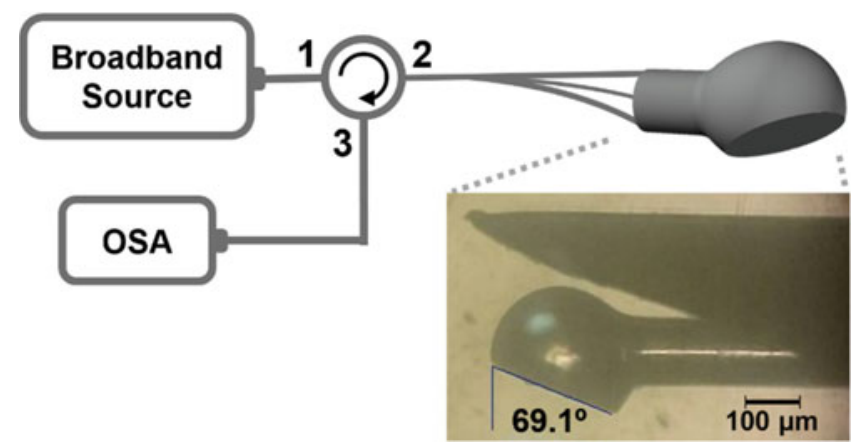

Fig. 1. Schematic of the experimental setup. The zoom shows a micrograph of the polished microsphere inside of a stainless steel needle.

cycle of the $\mathrm{CO}_{2}$ laser. In this case, the frequency, duty cycle, and the number of pulses used were $800 \mathrm{~Hz}, 50 \%$, and 4000, respectively.

With such parameters, a $270-\mu \mathrm{m}$-diameter microsphere was obtained. Asymmetry was introduced in the microsphere by polishing the structure at a desired angle. To do so, the microsphere was first inserted into a stainless steel needle (gauge 25) to protect the structure from bending during the polishing process. The whole pack was bounded with cyanoacrylate adhesive, which will be later on removed by dissolving it with acetone. The protected microsphere is then mounted on a protractor at a specified angle $\left(\theta_{1}\right)$, and the polishing process is performed. The polishing angle is given by $\pi / 2-\theta_{1}$. First, a polishing film with a grain size of $30 \mu \mathrm{m}$ was placed over a rubber polishing pad on top of a glass plate. The microsphere was polished in a figure-of-eight pattern with increasing pressure until the cross-section of the microsphere was flat at the desired angle. Then, fine polishing steps were executed using polishing films with decreasing grain sizes $(6,3$, and $1 \mu \mathrm{m})$. In the final polishing step, the microsphere performed three figure-of-eight patterns in a polishing film with grain size of $0.02 \mu \mathrm{m}$ mixed with four droplets of distilled water. The produced microsphere was polished at an angle of $70^{\circ}$, as shown in Fig. 1.

A schematic of the experimental setup is also depicted in Fig. 1. An erbium-doped broadband optical source, an optical circulator, and an optical spectrum analyzer (OSA) were used to obtain the reflection spectra. The broadband source had a central wavelength of $1550 \mathrm{~nm}$ (C-Band) and a bandwidth of $100 \mathrm{~nm}$, the optical circulator had a central wavelength also at $1550 \mathrm{~nm}$, and the OSA had a resolution of $35 \mathrm{pm}$. The reflected signal was previously normalized, taking as a reference the reflected signal from a cleaved single mode fiber in air (4\% Fresnel reflection).

The polished microsphere reflection spectra at two distinct temperatures are shown in Fig. 2(a). It is visible a phase change between the two reflection spectra due to thermal expansion of the sensing head. The asymmetry introduced in the microsphere generates several different reflections inside of the structure. The interference of the different optical paths produces an interferometric spectral modulation equivalent to the superposition of multiple two-wave interferometers. Although the reflection spectrum appears to be similar to a chaotic signal, it is just the result of the sum between the multiple interferometers. Fig. 2(b) presents the fast Fourier transform (FFT) of the two reflection spectra. The multiple peaks correspond to the different interferometric components, each one represented by the number of fringes $(\#) / \mathrm{nm}$.

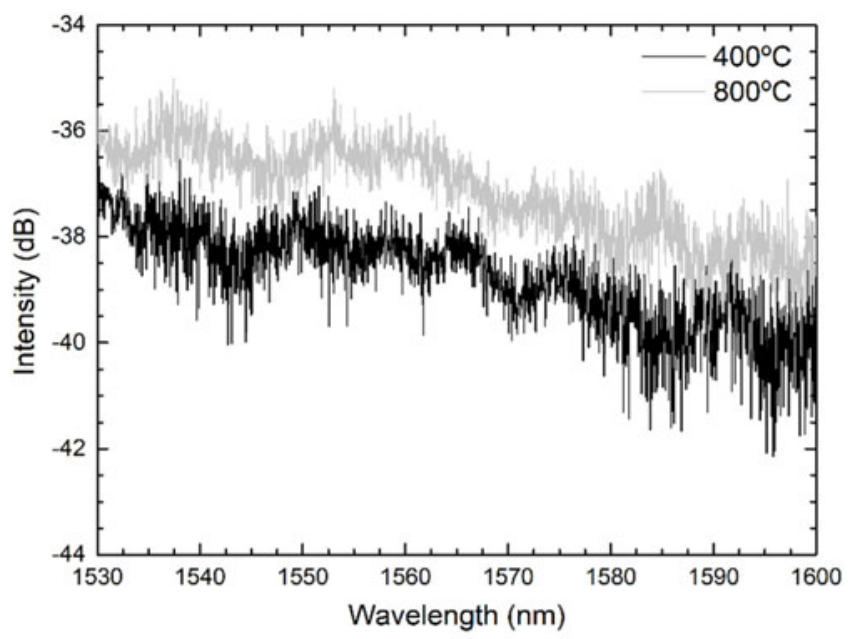

(a)

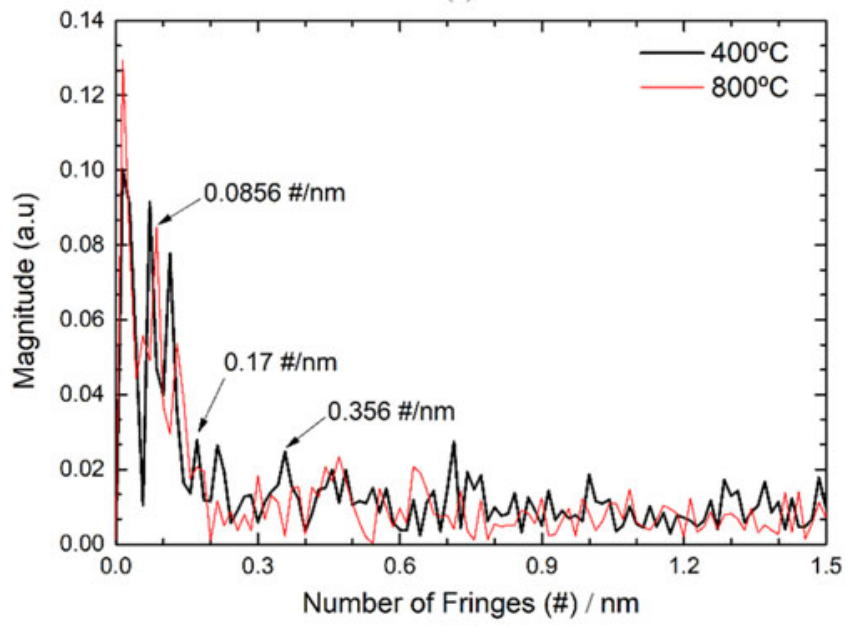

(b)

Fig. 2. (a) Reflection spectra of the polished microsphere at $400{ }^{\circ} \mathrm{C}$ and $800^{\circ} \mathrm{C}$. (b) FFT of the reflection spectra for both temperatures.

It is possible to separate the different interferometers by filtering each component out of the reflection spectrum. To obtain one component from the reflection spectrum, an FFT bandpass filter was applied with a central frequency equal to the peak frequency in the FFT and with a filter width given approximately by the width at the middle of the FFT peak (width at half maximum). Fig. 3 shows three different components with frequencies $0.0856,0.17$, and $0.356 \# / \mathrm{nm}$, at two distinct temperatures. The bandpass filter width used to filter each component was, respectively, 0.014, 0.016, and $0.020 \# / \mathrm{nm}$. The different optical paths inside of the structure change due to thermal expansion, resulting in a new reflection spectrum. Apart from the wavelength shift, the new reflection spectrum can be translated by different contributions (different intensities) of the multiple twowave interferometer components. This intensity exchange is visible in Fig. 3, where the different interferometric components have different intensities at distinct temperatures.

The structure was submitted to different temperatures, from $800{ }^{\circ} \mathrm{C}$ to $400{ }^{\circ} \mathrm{C}$. Two of the different interferometric components with 0.356 and $0.0856 \# / \mathrm{nm}$ were filtered and analyzed. For the higher frequency interferometer $(0.356 \# / \mathrm{nm})$, the obtained temperature sensitivity was $15.9 \pm 0.6 \mathrm{pm} /{ }^{\circ} \mathrm{C}$. As for the lower frequency interferometer $(0.0856$ $\# / \mathrm{nm}$ ), the sensitivity increased to $45 \pm 1 \mathrm{pm} /{ }^{\circ} \mathrm{C}$. 


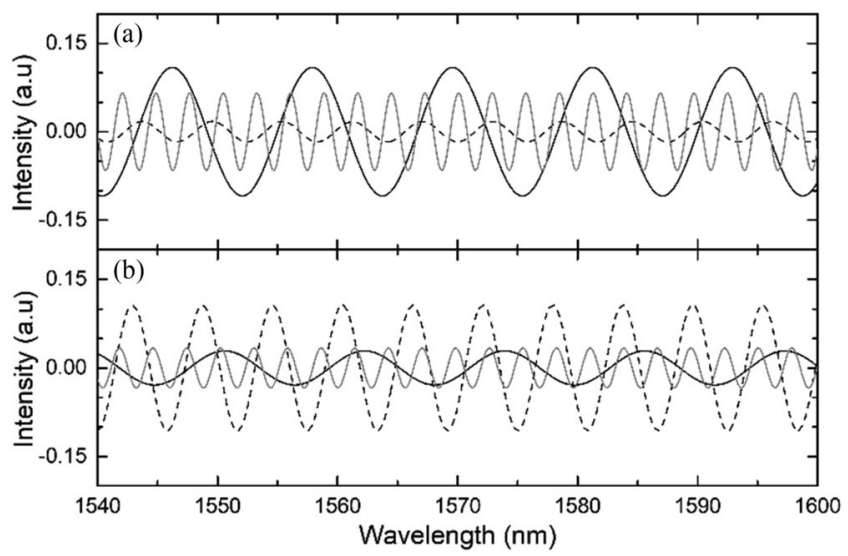

Fig. 3. (a) Filtered spectra at $700{ }^{\circ} \mathrm{C}$. (b) Filtered spectra at $800{ }^{\circ} \mathrm{C}$. Black line: cavity of $0.0856 \# / \mathrm{nm}$. Black dashed line: cavity of $0.17 \# / \mathrm{nm}$. Gray line: cavity of $0.356 \# / \mathrm{nm}$.

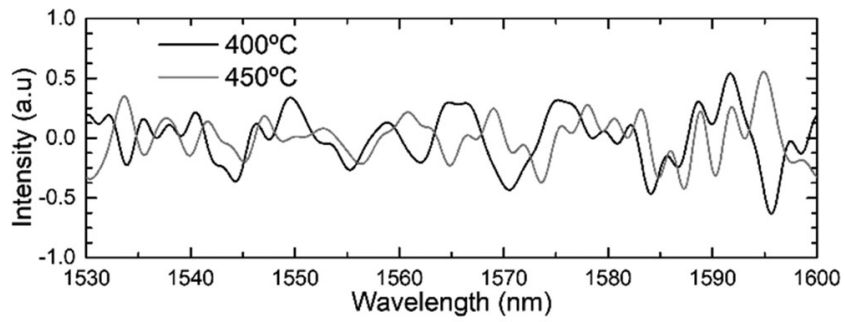

(a)

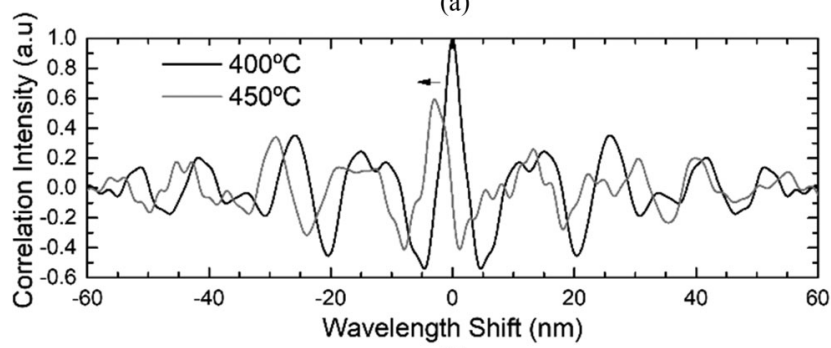

(b)

Fig. 4. (a) Filtered reflection spectra at two distinct temperatures $\left(400{ }^{\circ} \mathrm{C}\right.$ and $450{ }^{\circ} \mathrm{C}$ ). (b) Normalized autocorrelation (at $400{ }^{\circ} \mathrm{C}$ ) and cross-correlation of the reflection spectrum at a distinct temperature $\left(450^{\circ} \mathrm{C}\right)$.

A different way to obtain a response to temperature without filtering each component separately is to use a correlation method over the reflection spectra with the multiple interferometric components. The cross-correlation between two reflection spectra is calculated using the following equation [11]:

$$
R_{x y}(n)=\frac{1}{N} \sum_{k=0}^{N-1} x(k) \cdot y(n+k)
$$

where $N$ is the total sampling data points, $x(n)$ and $y(n)$ are the two spectral data, and $n$ is a value between $-(N-1)$ and $N-1$. Elements of $x(n)$ and $y(n)$ with negative indices or larger than $N-1$ are assumed as zero. If $x(n)$ and $y(n)$ are the same signal, the operation is called autocorrelation.

A bandpass filter was applied to the reflection spectra, selecting the interferometric components between 0.05 and $0.4 \# / \mathrm{nm}$. The filtered spectra at two distinct temperatures $\left(400{ }^{\circ} \mathrm{C}\right.$ and $\left.450{ }^{\circ} \mathrm{C}\right)$ are shown in Fig. 4(a). The reflection spectrum at $400{ }^{\circ} \mathrm{C}$ was used as

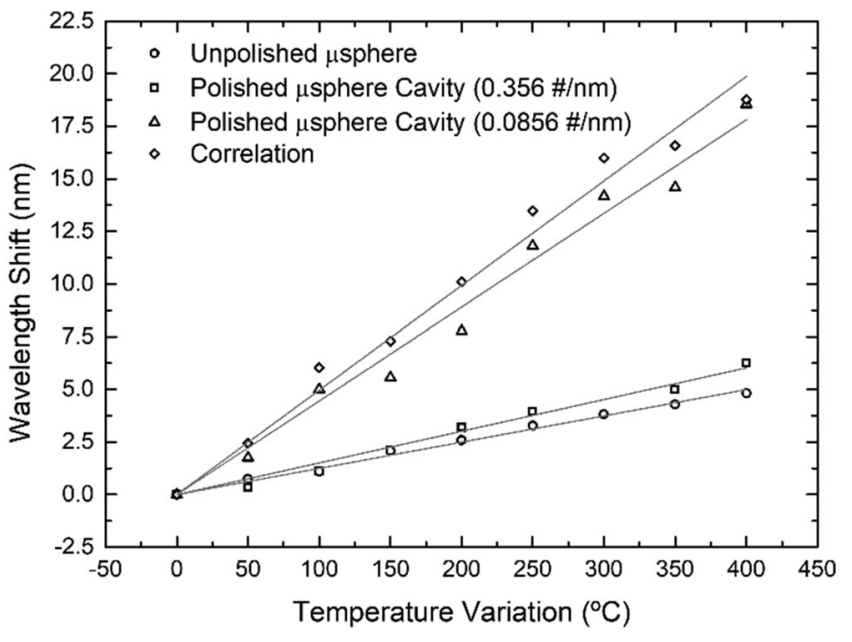

Fig. 5. Wavelength shift as a function of temperature variation for the unpolished microsphere, the two different filtered cavities of the polished microsphere $(0.0856 \# / \mathrm{nm}$ and $0.356 \# / \mathrm{nm})$, and for the correlation method.

TABLE 1. Comparison Between the Different Temperature Sensitivities.

\begin{tabular}{l|c}
\hline \hline & Temperature Sensitivity \\
\hline Unpolished $\mu$ sphere (electric arc) & $12.1 \pm 0.3 \mathrm{pm} /{ }^{\circ} \mathrm{C}$ \\
Polished $\mu$ sphere cavity $(0.356 \# / \mathrm{nm})$ & $15.9 \pm 0.6 \mathrm{pm} /{ }^{\circ} \mathrm{C}$ \\
Polished $\mu$ sphere cavity $(0.0856 \# / \mathrm{nm})$ & $45 \pm 1 \mathrm{pm} /{ }^{\circ} \mathrm{C}$ \\
Correlation Analysis & $50 \pm 1 \mathrm{pm} /{ }^{\circ} \mathrm{C}$ \\
\hline \hline
\end{tabular}

a reference and the autocorrelation was calculated. Then, the crosscorrelation was performed between the reflection spectra at different temperatures and the reference one. The autocorrelation and the cross-correlation spectra at a distinct temperature are depicted in Fig. 4(b). The correlation peak wavelength shift around $0 \mathrm{~nm}$ was monitored as a function of temperature variation. Note that the correlation wavelength shift is negative due to the reference temperature used in the correlation. The correct shift is in fact the symmetric value. A temperature sensitivity of $50 \pm 1 \mathrm{pm} /{ }^{\circ} \mathrm{C}$ was obtained with this method.

The wavelength shift as a function of temperature variation in relation to $400{ }^{\circ} \mathrm{C}$ for both filtered components of the polished microsphere, as well as for the correlation analysis, is depicted in Fig. 5, together with the result for the unpolished microsphere generated through electric arc. Table 1 summarizes the temperature sensitivity values for the different interferometric components and techniques used.

\section{CONCLUSION}

In conclusion, a multipath interferometer based on a polished multimode microsphere was demonstrated and applied for temperature sensing. Starting from a multimode fiber tip, a microsphere was produced but with no apparent improvements in terms of temperature sensitivity $\left(12.1 \pm 0.3 \mathrm{pm} /{ }^{\circ} \mathrm{C}\right)$. However, after introducing asymmetry by polishing the microsphere with a certain angle, multiple new optical paths are generated inside of the structure. These different optical paths produce an interferometric spectral modulation that can be seen as the superposition of multiple two-wave 
interferometers. Through signal processing, the different components can be extracted and monitored as a function of temperature. An enhancement of the temperature sensitivity was observed when considering interferometer components of lower frequency (higher free spectral range and, therefore, lower cavity length). A temperature sensitivity of $45 \pm 1 \mathrm{pm} /{ }^{\circ} \mathrm{C}$ was achieved for an interferometric component with $0.0856 \# / \mathrm{nm}$. Moreover, if a correlation method is used to obtain a response to temperature, an enhancement of the sensitivity is also verified. In such analysis there is no need to filter each interferometric component separately. A temperature sensitivity of $50 \pm 1$ $\mathrm{pm} /{ }^{\circ} \mathrm{C}$ was obtained using this method. In the future, a study should be done to see the influence of the polishing angle in the temperature sensitivity, in order to optimize the polishing angle for maximum sensitivity. An experiment should also be conducted in order to find the temperature for which saturation starts to occur. This will determine the maximum temperature for which the sensor response is still linear.

\section{ACKNOWLEDGMENT}

This work was supported by the Project NanoSTIMA-North Portugal Regional Operational Programme (NORTE-01-0145-FEDER-000016). The work of A. D. Gomes was supported by the Fundação para a Ciência e Tecnologia (FCT) (SFRH/BD/129428/2017).

\section{REFERENCES}

[1] Y. J. Rao, "Recent progress in fiber-optic extrinsic Fabry-Perot interferometric sensors," Opt. Fiber Technol., vol. 12, no. 3, pp. 227-237, 2006.

[2] M. S. Ferreira, L. Coelho, K. Schuster, J. Kobelke, J. L. Santos, and O. Frazão, "Fabry-Perot cavity based on a diaphragm-free hollow-core silica tube," Opt. Lett., vol. 36, no. 20, pp. 4029-4031, 2011.

[3] K. K. Chin et al., "Fabry-Perot diaphragm fiber-optic sensor," Appl. Opt., vol. 46, no. 31, pp. 7614-7619, 2007.

[4] R. M. André et al., "Simultaneous measurement of temperature and refractive index using focused ion beam milled Fabry-Perot cavities in optical fiber micro-tips,' vol. 24 , no. 13 , pp. 5764-5769, 2016.

[5] R. M. André et al., "Focused ion beam post-processing of optical fiber Fabry-Perot cavities for sensing applications," Opt. Exp., vol. 22, no. 11, pp. 13102-13108, 2014

[6] H. Sun, X. Zhang, L. Yuan, L. Zhou, X. Qiao, and M. Hu, "An optical fiber FabryPerot interferometer sensor for simultaneous measurement of relative humidity and temperature," IEEE Sensors J., vol. 15, no. 5, pp. 2891-2897, 2015.

[7] J. C. Knight, G. Cheung, F. Jacques, and T. A. Birks, "Phase-matched excitation of whispering-gallery-mode resonances by a fiber taper," Opt. Lett., vol. 22, no. 15, pp. 1129-1131, 1997.

[8] C. Monteiro, S. Silva, and O. Frazão, "Hollow microsphere Fabry-Perot cavity for sensing applications," IEEE Photon. Technol. Lett., vol. 29, no. 15, pp. 1229-1232, Aug. 2017.

[9] M. S. Ferreira, J. L. Santos, and O. Frazão, "Silica microspheres array strain sensor," Opt. Lett., vol. 39, no. 20, pp. 5937-5940, 2014.

[10] E. Li, X. Wang, and C. Zhang, "Fiber-optic temperature sensor based on interference of selective higher-order modes," Appl. Phys. Lett., vol. 89, no. 9, pp. 22-24, 2006.

[11] Y. Xu et al., "Optical fiber random grating-based multiparameter sensor," Opt. Lett., vol. 40, no. 23, pp. 5514-5517, 2015. 